\title{
Determinación de la tensión de laminación en caliente a partir de ensayos de tracción ${ }^{(\bullet)}$
}

\author{
A. Monsalve*, A. Artigas*, D. Celentano**, A. Guzmán*, R. Colás*** e Y. Houbaert****
}

Resumen

\begin{abstract}
La predicción del comportamiento mecánico de aceros laminados en caliente es un aspecto importante durante el procesamiento industrial. En el presente trabajo, las tensiones necesarias para el proceso de laminado, fueron estimadas usando tres métodos, el primero de los cuales se basa en la aplicación del modelo de Sellars, que toma en cuenta el coeficiente de fricción y el límite elástico. El segundo método, estima la tensión de laminado a partir de la tensión promedio obtenida durante los ensayos de tracción. El tercer método, estima la tensión de laminado a partir de los datos de potencia consumida por el equipo de laminación. Esta metodología fue aplicada en este estudio a un acero al carbono. La conclusión de este trabajo es que los resultados obtenidos por los métodos basados en el modelo de Sellars y de la potencia consumida, son coincidentes, en tanto que el método basado en la tensión de tracción, presenta valores ligeramente menores a los predichos por los otros modelos.
\end{abstract}

\section{Determination of the hot rolling stress by means of tensile tests}

\begin{abstract}
Prediction of mechanical behavior of hot rolled steels is an important aspect during industrial processing. The rolling stress needed to the process were estimated using three methods, the first of which is related to the Sellars`s model that takes into account the friction coefficient and the yield stress. The second method estimates the rolling stress as the average stress obtained during the tensile tests. The third method, estimates the rolling stress from the data of power consumed by the rolling equipment. This methodology was applied in this study to a carbon steel. The conclusion of the work is that results obtained by Sellars`s and consumed power, are coincident. The method based on the average tensile stress predicts stresses lower than those obtained with other two methods.
\end{abstract}

Keywords

Hot rolling; Modelling; Steels; Plastic deformation; Tensile test.

\section{INTRODUCCIÓN}

El laminado en caliente es una de las operaciones más usadas en la fabricación de acero. Por esta razón, la modelización del comportamiento mecánico de los materiales a alta temperatura es de importancia fundamental en la optimización de aquellos procesos de manufactura que involucran deformación en caliente ${ }^{[1]}$.

La relación entre los parámetros de proceso, las propiedades mecánicas del material y la tensión necesaria para laminación, ha sido descrita por Roberts y Lenard ${ }^{[2-4]}$. Básicamente, todos los modelos están basados en la relación general que se plantea en la teoría de la plasticidad[5]:

$$
\bar{\sigma}_{t}=\frac{2}{\sqrt{3}} \sigma_{0} F
$$

donde $\sigma_{0}$ es el límite elástico, $\bar{\sigma}_{t}$ es la tensión necesaria para laminar y $\mathrm{F}$ es una función que da cuenta de la influencia del coeficiente de fricción $\mu$ y de un factor de forma $\Delta$, asociado a la zona de deformación, esto es:

$$
\mathrm{F}=\mathrm{F}(\mu, \Delta)
$$

(•) Trabajo recibido el día 2 de abril de 2012 y aceptado en su forma final el día 7 de junio de 2012.

* Universidad de Santiago de Chile, Dpto. Ingeniería Metalúrgica, Av. L. B. O’Higgins 3363, Estación Central. Santiago de Chile.

** Departamento de Ingeniería Mecánica y Metalúrgica, Pontificia Universidad Católica de Chile.

*** Facultad de Ingeniería Mecánica y Eléctrica, Universidad Autónoma de Nuevo León, 66451 San Nicolás de los Garza, N.L. México.

**** Department of Materials Science and Engineering, University of Ghent, Bélgica. 
en que $\Delta$ se relaciona con las características geométricas del proceso de deformación, tal como el espesor promedio del producto laminado y la longitud de contacto entre los rodillos.

Se han llevado a cabo numerosos estudios para predecir la fuerza de laminado en procesos de laminación. Wray ${ }^{[6]}$ desarrolló un modelo para predecir el valor de la fuerza de laminado en el tren laminador final de un proceso de laminación en caliente. El estudio está basado, en un modelo simple y los datos de resistencia a la fluencia se obtienen a partir de experimentos de laboratorio. El modelo toma en consideración la fuerza de separación de los rodillos $\mathrm{P}$, que depende de la longitud de laminación (L), la resistencia media efectiva a la deformación $(\overline{\mathrm{k}})$ y un factor geométrico $Q$, a través de:

$$
P=\bar{k} L Q
$$

A su vez, la resistencia efectiva promedio a la deformación, se calcula a partir de la deformación $\varepsilon$, la velocidad de deformación $\dot{\varepsilon}$, la temperatura absoluta $\mathrm{T}$ y un conjunto de constantes $\left(\sigma_{0}, \mathrm{~K}, \mathrm{n}, \mathrm{m}, \mathrm{C}\right)$ que dependen del material a partir de:

$\overline{\mathrm{k}}=\frac{2}{\sqrt{3}}\left\{\sigma_{0}+\mathrm{K}\left[\frac{2}{\sqrt{3}}\left(\varepsilon-\varepsilon_{0}\right)\right]\right\}^{\mathrm{n}}\left(\frac{2}{\sqrt{3}} \dot{\varepsilon}\right)^{\mathrm{m}} \exp (-\mathrm{CT})(4)$

En el caso de ese estudio fueron determinadas para un acero 0,03\% C; 1,4\% Mn; 0,23\% Si; 0,11\% Nb. Para obtener la dependencia de la velocidad de deformación con la deformación, Wray tomó en cuenta la velocidad lineal de la banda a la entrada de los rodillos $\mathrm{V}_{\mathrm{s}}$, el valor de la deformación $\varepsilon$, el radio del rodillo $\mathrm{R}$, la fracción de reducción de espesor de la banda $\mathrm{r}$ y el valor del espesor de la banda a la entrada $h_{0}$.

$$
\grave{\varepsilon}=\frac{2 \mathrm{~V}_{\mathrm{s}} \exp (\varepsilon)}{\mathrm{h}_{0}}\left\{1-\left[1-\frac{\mathrm{h}_{0}(\mathrm{r}-1)+\exp (-\varepsilon)}{2 \mathrm{R}}\right]^{2}\right\}^{1 / 2}
$$

Uno de los parámetros más importantes del modelo es la longitud del arco de contacto proyectada sobre el plano medio de la banda, que depende del espesor de dicha banda a la entrada, del radio del rodillo y de los espesores inicial y final de la banda. Los resultados del modelo incluyen consideraciones acerca de la recristalización estática producida entre pasadas. Asimismo los resultados del modelo propuesto por Wray, se ajustan bien a los resultados experimentales, tomando en consideración que en algunos casos se produce recristalización dinámica, durante los períodos entre pasadas. Este procedimiento planteado por Wray requiere del conocimiento de al menos seis constantes del material, lo cual constituye una desventaja.
Otras contribuciones importantes son los trabajos de Sims ${ }^{[7]}$, relacionados con el cálculo de las fuerzas y del torque de laminado en rodillos de laminación en caliente; el trabajo de Ford ${ }^{[8]}$ relativo a un cálculo simplificado de las tensiones de laminación y el trabajo de Kokado ${ }^{[9]}$, relacionado con las predicciones de tensión de laminación en rodillos de laminación en caliente. Béjar ${ }^{[10]}$ desarrolló un modelo matemático de la curva tensión-deformación para austenita en un acero C-Mn, basado en ensayos de torsión, obteniendo resultados que se ajustan razonablemente a las curvas experimentales. Las ecuaciones constitutivas toman en consideración los mecanismos gobernantes durante la deformación plástica, responsable de la forma de la curva. El modelo deducido utiliza las variables de proceso y la composición química del acero y está basado en una correlación estadística entre los diversos parámetros de dicho modelo. Las metodologías planteadas en el presente trabajo para estimar las tensiones de laminado, no requieren del conocimiento de tantas constantes como lo que propone Wray; tampoco incorpora valores de variables de proceso, como Ford y Kokado; ni realiza un análisis fenomenológico y estadístico como el planteado por Béjar. La contribución del presente trabajo radica en la simplicidad de la metodología propuesta, especialmente los métodos de la tensión promedio de tracción y el de la potencia consumida.

En general, el mejor método para determinar las tensiones de laminación consiste en realizar ensayos de compresión y aplicar un análisis inverso para la interpretación de los resultados. El análisis inverso fue propuesto en la década de los 90 por Gelin ${ }^{[11]}$ y Gavrius $^{[12]}$ siendo desarrolladas recientemente soluciones más avanzadas por Boyer ${ }^{[13]}$, Forestier ${ }^{[14]}$ y Szeliga ${ }^{[15]}$, pasando este método a ser el procedimiento estándar para la solución al problema de determinar las tensiones de laminación. Finalmente, cabe señalar que Szeliga ${ }^{[16]}$ aplicó el análisis inverso para conocer las tensiones en un ensayo de tracción. Actualmente, se acostumbra a utilizar modelos de elementos finitos con el fin de simular procesos de laminación, los cuales requieren del conocimiento del valor del límite elástico del material en las condiciones en que se realiza la laminación en caliente. En el método de análisis inverso se requiere conocer el valor de cantidades experimentales como la fuerza de laminación y la geometría, obteniéndose los valores del coeficiente de fricción en la intercara además de los coeficientes de las ecuaciones de los modelos. Si $\mathrm{P}_{1}, \mathrm{P}_{2} \ldots \mathrm{P}_{\mathrm{r}}$ son los parámetros de un determinado modelo (por ejemplo los parámetros del ajuste de Hollomon, el índice de sensibilidad a la velocidad de deformación etc.), se construye una función objetivo que permita encontrar los valores de estos parámetros que minimicen la dife- 
rencia entre los valores experimentales de una variable $\mathrm{M}_{\text {exp }} \mathrm{y}$ aquéllos calculados $\mathrm{M}_{\text {calc }}$, utilizando la técnica de mínimos cuadrados. Una vez más, la propuesta del presente trabajo se destaca por la simplicidad en comparación con estos métodos, debido a la necesidad que presenta el método del análisis inverso de un respaldo computacional importante en cuanto a software especializado y a potencia de cálculo.

En el presente trabajo, las tensiones necesarias para el proceso de laminación, fueron estimadas usando tres métodos, los cuales se basan en medidas de laboratorio y en modelos fenomenológicos, constituyendo una aproximación simplificada a los métodos basados en el análisis inverso y otros desarrollados. La originalidad del presente trabajo radica en que a partir de ensayos de tracción se estudia predecir la tensión necesaria para producir la laminación, lo cual resulta interesante, teniendo en cuenta que las velocidades de deformación en los procesos de laminación son mayores que en los ensayos de tracción. Además, el proceso de laminación ocurre en condiciones de deformación biaxial y tensiones compresivas, en tanto que el ensayo de tracción es de deformación triaxial $\mathrm{y}$ tensiones tractivas.

Cabe destacar que la tensión de laminación no fue medida directamente, estimándose esta tensión de laminación a partir de la potencia consumida por el equipo de laminación, tomando en consideración que la fricción en los cojinetes, la eficiencia del motor y el rozamiento en los engranajes se incluyen dentro de un factor de corrección. Para esto, se midió la potencia consumida por el laminador a través de un analizador de potencia, pudiendo determinarse en todo momento la potencia consumida durante el proceso de laminación.

\subsection{Método basado en el modelo de Sellars}

El modelo usado en este trabajo puede ser descrito por medio de la tensión $\bar{\sigma}_{t}$ necesaria para laminar, la cual puede ser calculada a partir de $\mathrm{e}^{[5]}$.

$$
\bar{\sigma}_{t}=\frac{2}{\sqrt{3}} \sigma_{0}\left[\frac{1}{Q}(\exp (Q)-1)\right]
$$

donde $\sigma_{0}$ es el límite elástico del material y el término $Q$ se calcula a partir de:

$$
Q=\frac{\mu L}{\bar{h}}
$$

donde $\mu$ es el coeficiente de fricción que se supone igual a $0,17^{[17-19]}$, L corresponde a la longitud de contacto entre los rodillos y el material laminado pudiendo ser calculado a partir de:

$$
L=\left(R \Delta h-\frac{\Delta h^{2}}{4}\right)^{0,5}
$$

donde $\Delta \mathrm{h}$ corresponde a la reducción de espesor y $\mathrm{R}$ al radio de los rodillos. El término $\bar{h}$, corresponde al espesor promedio de la banda entre la entrada y la salida.

El valor del límite elástico promedio a una temperatura y velocidad de deformación dada, puede ser obtenido a partir de un modelo propuesto por Sellars ${ }^{[19}$ y 20$]$, que relaciona el límite elástico con la temperatura y la velocidad de deformación a partir de:

$$
A\left(\operatorname{senh}\left(\alpha \sigma_{0}\right)\right)^{n}=\dot{\varepsilon} \exp \left(\frac{Q_{\text {act }}}{R T}\right)=Z
$$

donde $\mathrm{A}$ es un parámetro que depende únicamente del material, $\alpha$ depende del material y $\sigma_{0}$ es el límite elástico a la temperatura absoluta $\mathrm{T}$ y velocidad de deformación $\grave{\varepsilon}$ usada en el proceso, $\mathrm{Q}_{\mathrm{act}}$ corresponde a la energía de activación requerida para deformación plástica, $\mathrm{R}$ es la constante universal de los gases y $\mathrm{Z}$ es el parámetro Zener-Hollomon.

La velocidad de deformación producida por los rodillos fue calculada usando ${ }^{[5]}$ :

$$
\overline{\dot{\varepsilon}}=\frac{v}{\sqrt{R \Delta h}} \ln \frac{h_{0}}{h_{f}}
$$

con $\mathrm{v}=\omega \mathrm{R}$, siendo $\omega$ la velocidad angular del rodillo; $h_{0} y_{f}$ son los espesores inicial y final de la banda laminada.

\subsection{Método de ensayo de tracción}

La tensión promedio obtenida a partir de ensayos de tracción, $\bar{\sigma}_{\mathrm{t}}$ puede ser calculada a partir de:

$$
\bar{\sigma}_{t}=\frac{1}{\varepsilon_{f}-\varepsilon_{0}} \int_{\varepsilon_{0}}^{\varepsilon_{f}} \sigma d \varepsilon
$$

donde $\varepsilon_{0}$ y $\varepsilon_{\mathrm{f}}$, son respectivamente la deformación al inicio y al final del ensayo de tracción considerado (usualmente $\varepsilon_{0}=0$ ). 


\subsection{Método de potencia consumida}

Es posible medir la potencia consumida por el laminador y calcular la tensión $\bar{\sigma}_{t}$ requerida para laminar la banda, a partir de ${ }^{[5}$ y 21]:

$$
\bar{\sigma}_{t}=\frac{P_{\text {req }}}{\pi L^{2} w \omega}
$$

donde $\mathrm{P}_{\text {req }}$ corresponde a la potencia requerida para el laminado, la cual es calculada midiendo la corriente y el voltaje consumido por el equipo durante la laminación en caliente, w es el ancho de la banda y $\omega$ es la velocidad angular de los rodillos. Experimentalmente, fue posible determinar que $\mathrm{P}_{\text {req }}$ puede ser calculada a partir de la potencia consumida $\mathrm{P}_{\text {con }}$, usando:

$$
P_{\text {req }}=\frac{P_{\text {con }}}{2,6}
$$

El factor 2,6 fue usado en todos los casos estudiados, y es independiente de la temperatura y la velocidad de deformación. Este factor, resume el efecto de diferentes parámetros tales como, la fricción en los cojinetes, la pérdida de potencia en los engranajes, la eficiencia en el motor y fue determinado experimentalmente. Asimismo este factor fue medido para el equipo utilizado en el presente trabajo, un laminador Jolliot de $200 \mathrm{~mm}$ de ancho de rodillos, debiendo ser calculado nuevamente al utilizar otro equipo de laminación. Para esto se determinó para varios aceros, la potencia consumida por el laminador utilizando un analizador de potencia, después de lo cual se estimó la tensión de laminación con ayuda de la Ec. (12), comparándose esta tensión con las estimaciones obtenidas por los ensayos de tracción y por el modelo de Sellars. Esto permitió deducir que sólo una parte de la potencia consumida se invertía realmente en deformar el metal, encontrándose que la relación entre ambas potencias era de 2,6.

En el presente trabajo, el proceso de laminación en caliente, se modelizó utilizando parámetros obtenidos a partir de ensayos de tracción llevados a cabo a diferentes temperaturas y diferentes velocidades de deformación. El material estudiado fue un acero SAE 1025. A partir de estos datos, se utilizó un modelo matemático que toma en consideración el parámetro de Zener-Hollomon, con el fin de calcular el límite elástico a diferentes temperaturas y distintas velocidades de deformación, a partir de lo cual se calcula la tensión de laminado. Los resultados de este método se compararon con los valores de tensión promedio obtenidos a partir de ensayos de tracción realizados a diferentes velocidades de deformación. Finalmente, la tensión de laminación fue estimada indirectamente a partir de la potencia consumida por el laminador. El objetivo final del trabajo es estimar la tensión de laminación en un proceso de laminado mediante tres métodos y comparar los resultados obtenidos con el fin de validar entre sí dichos procedimientos.

\section{PROCEDIMIENTO EXPERIMENTAL}

En el presente trabajo se estudió el acero SAE 1025, cuya composición química se muestra en la tabla I.

Tabla I. Composición química del acero

Table I. Chemical composition of the steel

\begin{tabular}{lccccc}
\hline Elemento & $\mathbf{C}$ & Mn & Si & $\mathbf{P}$ & $\mathbf{S}$ \\
\hline$\%$ peso & 0,25 & 1,06 & 0,22 & 0,017 & 0,028 \\
\hline
\end{tabular}

Las propiedades mecánicas de este acero se muestran en la tabla II.

La microestructura de este acero consiste en ferrita y perlita, como se muestra en la figura 1 .

Se usaron probetas cilíndricas de 9,5 $\mathrm{mm}$ de diámetro y $100 \mathrm{~mm}$ de longitud, de acuerdo con la norma ASTM E-21 [22]. Se ensayaron siete temperaturas: $600-650-700-750-800-900$ y $950^{\circ} \mathrm{C}$. Los ensayos de tracción fueron realizados bajo control de des- plazamiento, usando tres velocidades de desplazamiento de cruceta: 1,10 y $70 \mathrm{~mm} / \mathrm{min}$, correspondientes a velocidades iniciales de deformación de $10^{-4}, 1 \times 10^{-3}$ y $7 \times 10^{-3}\left(\mathrm{~s}^{-1}\right)$. Sin embargo, debido al acomodamiento de las mordazas y a que no se utilizó extensómetro por las altas temperaturas a las que se ensayó, las velocidades de deformación medidas a partir de la pendiente de la curva deformación versus tiempo fueron: $10^{-4} ; 1,2 \times 10^{-3}$ y $8,6 \times 10^{-3}\left(\mathrm{~s}^{-1}\right)$. 
DETERMINACIÓN DE LA TENSIÓN DE LAMINACIÓN EN CALIENTE A PARTIR DE ENSAYOS DE TRACCIÓN DETERMINATION OF THE HOT ROLLING STRESS BY MEANS OF TENSILE TESTS

Tabla II. Propiedades mecánicas del acero medidas a temperatura ambiente

Table II. Mechanical properties of the steel measured at room temperature

\begin{tabular}{ccccccc}
\hline $\begin{array}{c}\text { Límite } \\
\text { elástico } \\
(\mathrm{MPa})\end{array}$ & $\begin{array}{c}\text { UTS } \\
(\mathrm{MPa})\end{array}$ & $\begin{array}{c}\text { Elongación } \\
\text { total }(\%)\end{array}$ & $\begin{array}{c}\text { Reducción } \\
\text { de área } \\
(\%)\end{array}$ & $\begin{array}{c}\text { Dureza } \\
\text { Brinell }\end{array}$ & $\begin{array}{c}\mathbf{k} \text { (coeficiente } \\
\text { de Hollomon } \\
\sigma=k \varepsilon \text { n) }(\mathrm{MPa})\end{array}$ & $\begin{array}{c}\mathrm{n} \text { (índice } \\
\text { endurecimiento } \\
\text { por deformación) }\end{array}$ \\
\hline 434 & 587 & 24 & 65 & 90 & 374 & 0,23 \\
\hline
\end{tabular}

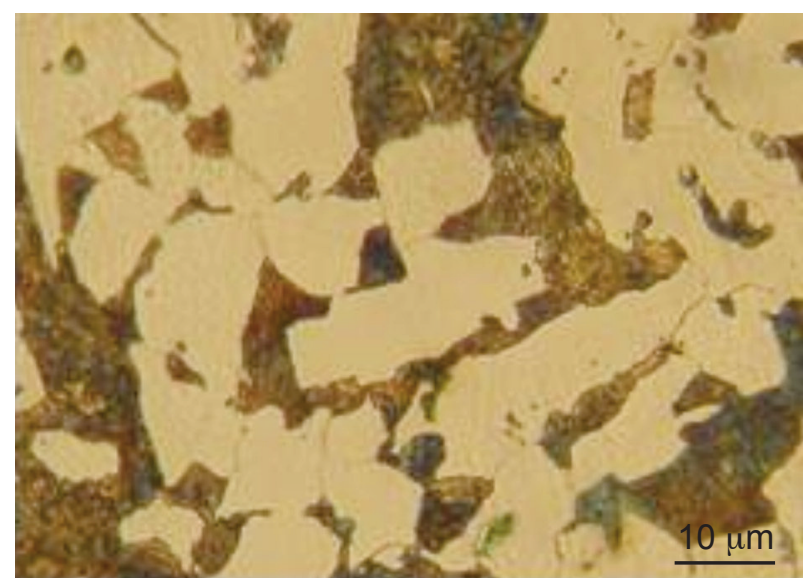

Figura 1. Microestructura del acero estudiado, consistente en ferrita y perlita.

Figure 1. Microstructure of the steel studied, consisting in ferrite plus pearlite.

Se usaron muestras de $8,1 \mathrm{~mm}$ de espesor, $8,1 \mathrm{~mm}$ de ancho, y $125 \mathrm{~mm}$ de longitud que fueron laminadas en caliente. Se maquinó una cuña de $30^{\circ}$ en el extremo de las probetas para facilitar el contacto con los rodillos. Las muestras para laminación fueron homogeneizadas durante $20 \mathrm{~min}$ a la temperatura del ensayo, siendo las deformaciones medidas de 10,20 y $30 \%$. La potencia necesaria para laminar las muestras fue medida en cada ensayo. El diámetro de los rodillos y la velocidad de rotación fueron respectivamente de $125,5 \mathrm{~mm}$ y $26 \mathrm{rpm}$.

Las muestras usadas para los ensayos de tracción se muestran en la figura 2 a) y las muestras usadas para laminación se muestran en la figura 2 b).

Para los ensayos de tracción cada muestra se introdujo en un horno. Después de esto, se encendió el horno hasta alcanzar la temperatura del ensayo. La muestra se mantuvo a esta temperatura durante $20 \mathrm{~min}$ con el fin de calentarlas homogéneamente hasta la temperatura de ensayo, después de lo cual comienza el ensayo de tracción. Además, en el caso de ensayos de laminación, las muestras fueron homogeneizadas durante $20 \mathrm{~min}$ a las temperaturas de laminación antes de cada ensayo, siendo enfriadas en aire al final de cada experiencia.

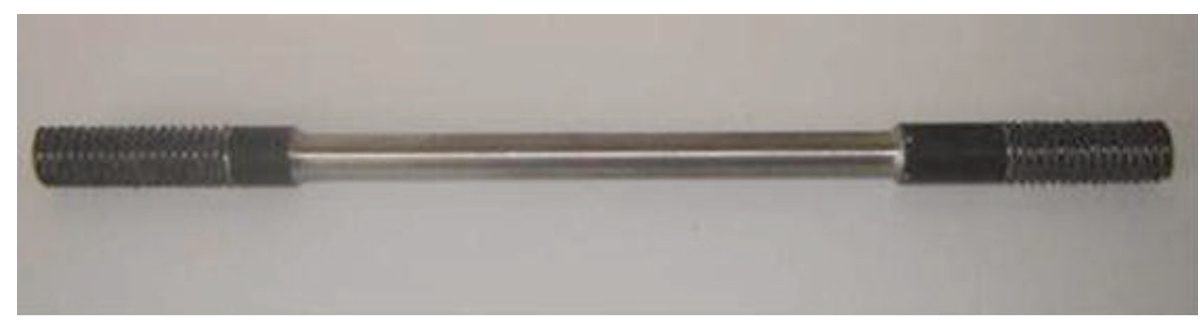

(a)

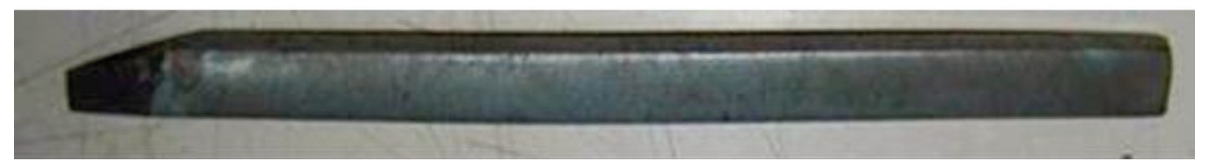

(b)

Figura 2. (a) Muestra usada para los ensayos de tracción; (b) Muestra usada para los ensayos de laminado.

Figure 2. (a) Sample used for tensile tests; (b) Sample used for rolling tests. 


\section{RESULTADOS}

La microestructura original del acero fue ferrítico-perlítica, como puede observarse en la figura 1 . Después de los ensayos de tracción a 600,650 y $700{ }^{\circ} \mathrm{C}$ comienza la globulización de la perlita, apareciendo una microestructura típica consistente en perlita fina de forma esférica en una matriz ferrítica. Debido a las altas temperaturas de ensayo, las muestras estudiadas, muestran menor resistencia mecánica que aquéllas ensayadas a temperaturas menores. A $750^{\circ} \mathrm{C}$, el ensayo se lleva a cabo en un material con estructura bifásica (ferrita más austenita), obteniéndose un comportamiento mecánico que es función de las fases presentes. Para los tratamientos realizados sobre $800^{\circ} \mathrm{C}$, los ensayos se llevaron a cabo en austenita, obteniéndose una menor resistencia mecánica.

La figura 3 a) muestra las curvas tensión-deformación obtenidas para cada una de las siete temperaturas a una velocidad de deformación de $1 \times 10^{-4}\left(\mathrm{~s}^{-1}\right)$.

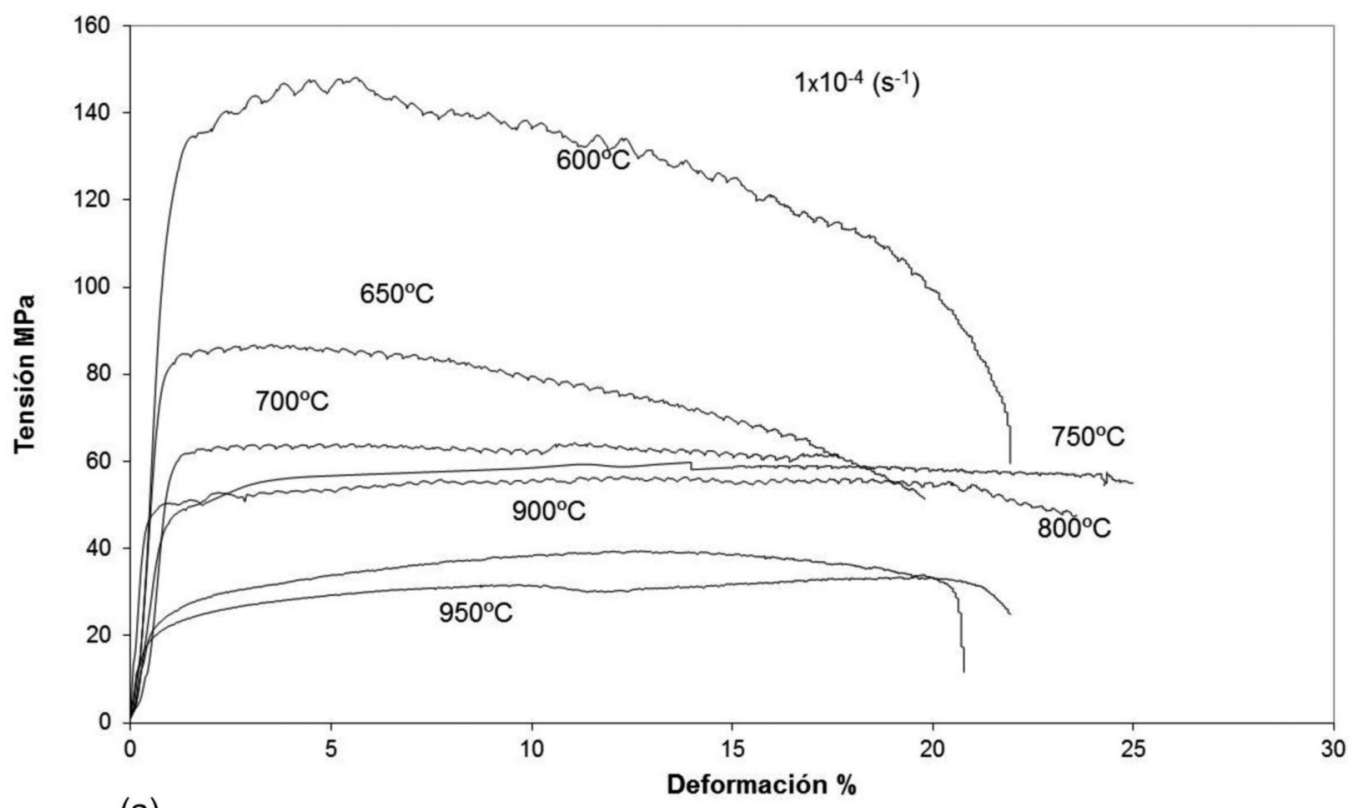

(a)

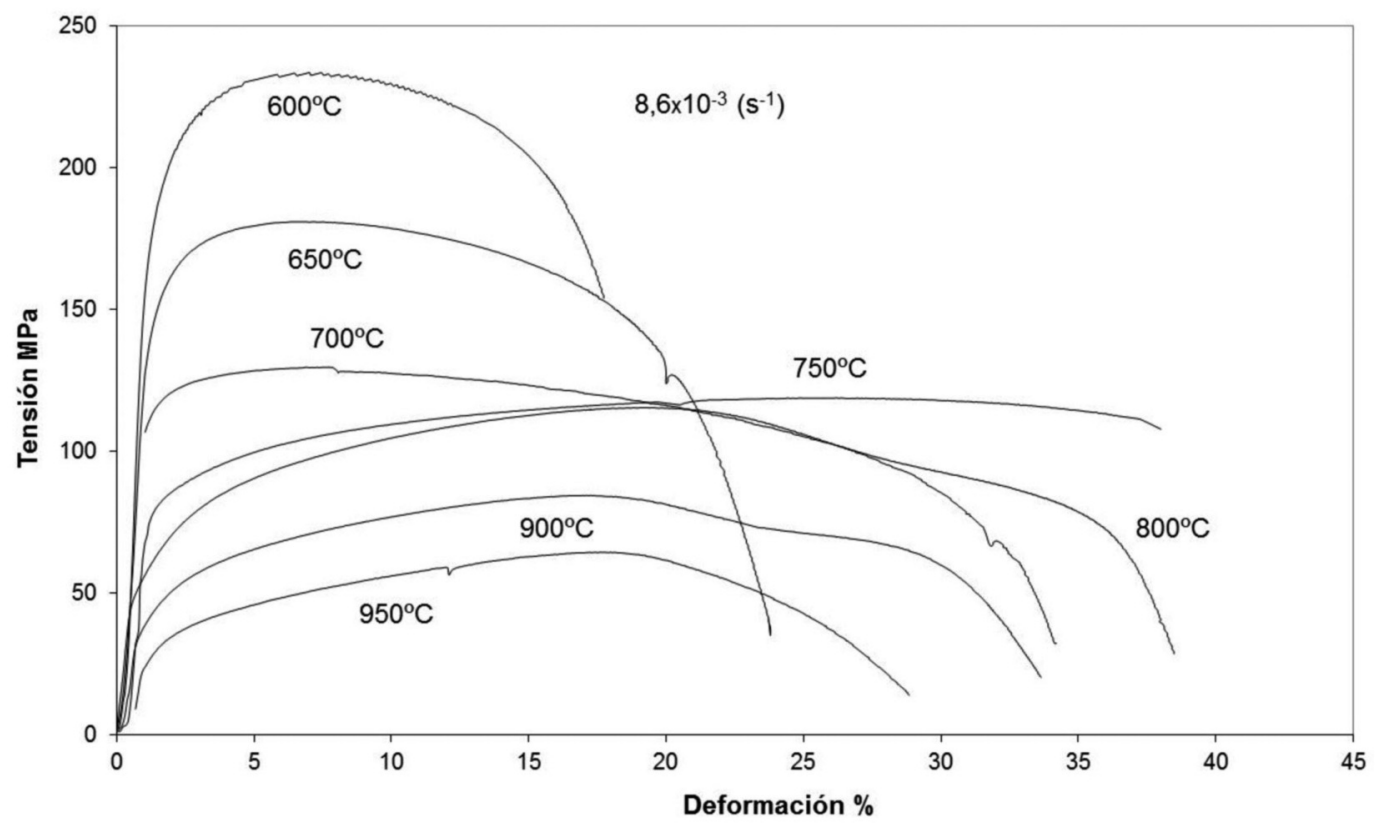

(b)

Figura 3. Ensayos de tracción (a) velocidad de deformación $1 \times 10^{-4}\left(\mathrm{~s}^{-1}\right)$; (b) velocidad de deformación 0,0086 $\left(\mathrm{s}^{-1}\right)$.

Figure 3. Tensile tests (a) strain rate $1 \times 10^{-4}\left(\mathrm{~s}^{-1}\right)$; (b) strain rate $0.0086\left(\mathrm{~s}^{-1}\right)$. 
Se puede observar que las curvas muestran dos formas diferentes: aquellas curvas que corresponden a ensayos sobre $750^{\circ} \mathrm{C}$ desarrollan un estado estacionario, mientras que aquéllas ensayadas por debajo de $750^{\circ} \mathrm{C}$ alcanzan un máximo y luego, la tensión disminuye. En el caso de una temperatura menor de $750^{\circ} \mathrm{C}$, la deformación ocurre en una microestructura ferrítica y para temperaturas mayores de $750^{\circ} \mathrm{C}$, la deformación ocurre en austenita. El descenso en la tensión para las curvas de bajas temperaturas y la aparición de una tensión aproximadamente estacionaria para ensayos realizados a altas temperaturas, puede explicarse por la competencia entre el endurecimiento por deformación y el ablandamiento debido a la recristalización dinámica. A bajas temperaturas, predomina la recristalización dinámica sobre el endurecimiento por deformación, ocurriendo lo contrario a altas temperaturas.

Los resultados correspondientes a una velocidad de deformación de $8,6 \times 10^{-3}\left(\mathrm{~s}^{-1}\right)$ se muestran en la figura $3 \mathrm{~b}$ ). Como en el caso previo, para temperaturas menores de $750^{\circ} \mathrm{C}$, las curvas muestran un máximo donde el incremento en la resistencia se relaciona con el endurecimiento por deformación y la disminución en la resistencia se relaciona con la recristalización dinámica. Los valores de la resistencia mecánica son mayores en este caso en comparación con aquéllos de la figura 3 a) debido a la mayor velocidad de deformación.

Además, en el caso de temperaturas más altas, es posible encontrar un mayor tamaño de grano debido al crecimiento de grano durante los ensayos.

La figura 4 muestra los ensayos de tracción a tres velocidades de deformación a 600 y $900^{\circ} \mathrm{C}$. En ambas curvas se observa una disminución en la resistencia mecánica y un aumento en la elongación total con el incremento de temperatura, así como también el incremento en la resistencia mecánica con la velocidad de deformación. En aquellos casos correspondientes a temperaturas por debajo de $750^{\circ} \mathrm{C}$, el incremento en la resistencia mecánica se relaciona con la microestructura y también a las menores temperaturas. A temperatura sobre $700^{\circ} \mathrm{C}$, los procesos de deformación se llevan a cabo en una microestructura austenítica, produciendo menores resistencias mecánicas y mayores elongaciones totales en comparación con aquellos valores obtenidos a menores temperaturas.

Los datos obtenidos a partir de las curvas tensióndeformación, fueron usados para determinar la dependencia del límite elástico respecto del parámetro $Z$. Con el fin de ajustar los datos experimentales se supuso un valor de $312 \mathrm{~kJ} / \mathrm{mol}{ }^{[23}$ y 24] para $Q$ y de 0,011875 para $\alpha^{[25]}$ (Fig. 5). Este valor de $\alpha$, fue propuesto por Urcola para aceros C-Mn y fue aplicado en este trabajo para el acero estudiado, debido a que la composición química de ambos aceros es suficientemente similar.

Las temperaturas $A_{1}$ (temperatura eutectoide) y $\mathrm{A}_{3}$ (máxima temperatura de equilibrio $\gamma / \alpha$ para la composición del acero estudiado), fueron calculadas usando las siguientes ecuaciones:

$$
\begin{gathered}
\mathrm{A}_{1}\left({ }^{\circ} \mathrm{C}\right)=723-10,7(\% \mathrm{Mn})-16,9(\% \mathrm{Ni})+ \\
29,1(\% \mathrm{Si})+16,9(\% \mathrm{Cr})+6,38(\% \mathrm{~W})+ \\
290(\% \mathrm{As})
\end{gathered}
$$

$$
\begin{gathered}
\mathrm{A}_{3}\left({ }^{\circ} \mathrm{C}\right)=910-203(\% \mathrm{C})^{0,5}-15,2(\% \mathrm{Ni})+ \\
44,7(\% \mathrm{Si})+104(\% \mathrm{~V})+31,5(\% \mathrm{Mo})+ \\
13,1(\% \mathrm{~W})-30(\% \mathrm{Mn})-11(\% \mathrm{Cr})-20(\% \mathrm{Cu}) \\
+700(\% \mathrm{P})+120(\% \mathrm{As})
\end{gathered}
$$

que fueron propuestas por Andrews ${ }^{[1]}$. Estas ecuaciones dieron $\mathrm{A}_{1}=728^{\circ} \mathrm{C}$ y $\mathrm{A}_{3}=800^{\circ} \mathrm{C}$. Por esta razón los resultados fueron divididos en dos grupos de acuerdo a las temperaturas: menor o igual a $728^{\circ} \mathrm{C}$ (estructura ferrítica) y mayores de $800^{\circ} \mathrm{C}$, con una estructura austenítica. En ambos casos, se graficó $\ln Z$ versus $\operatorname{lnsenh}\left(\alpha \sigma_{0}\right)$, obteniendo dos líneas rectas. Los mejores ajustes para ambos casos se muestran en la figura 5 y corresponden a:

$$
\begin{gathered}
\ln Z=5,1868 \operatorname{Insenh}\left(\alpha \sigma_{0}\right)+30,125 \\
T \leq 728^{\circ} \mathrm{C} \\
\ln Z=7,5968 \operatorname{Insenh}\left(\alpha \sigma_{0}\right)+31,723 \\
T>800^{\circ} \mathrm{C}
\end{gathered}
$$

los cuales permiten calcular el límite elástico a cualquier temperatura y velocidad de deformación, y serán usados en la evaluación de las tensiones de laminación. En ambos casos se ha supuesto $\alpha=0,011875$. Para temperaturas entre 728 y $800^{\circ} \mathrm{C}$, la microestructura corresponde a ferrita y austenita, por tanto a cada temperatura, el cálculo de $\ln Z$ considera la predicción de las ecuaciones (16) y (17) de acuerdo con la regla de la palanca aplicada en el diagrama de equilibrio. Esto significa que para la zona bifásica, el cálculo del límite elástico se considera como un valor obtenido a partir de una suma ponderada de los límites elásticos obtenidos para la ferrita y para la austenita, con factores de ponderación correspondientes a las fracciones de cada fase, de acuerdo al diagrama de equilibrio. Para el caso de una microestructura consistente en dos fases, se pueden aplicar modelos más sofisticados con el fin de estimar el valor de $\ln Z$, tal como aquéllos que toman en cuenta un modelo tipo Avrami para la transformación de ferrita a austenita $^{[26]}$. Para la ferrita (Ec. (16)), el valor del pará- 

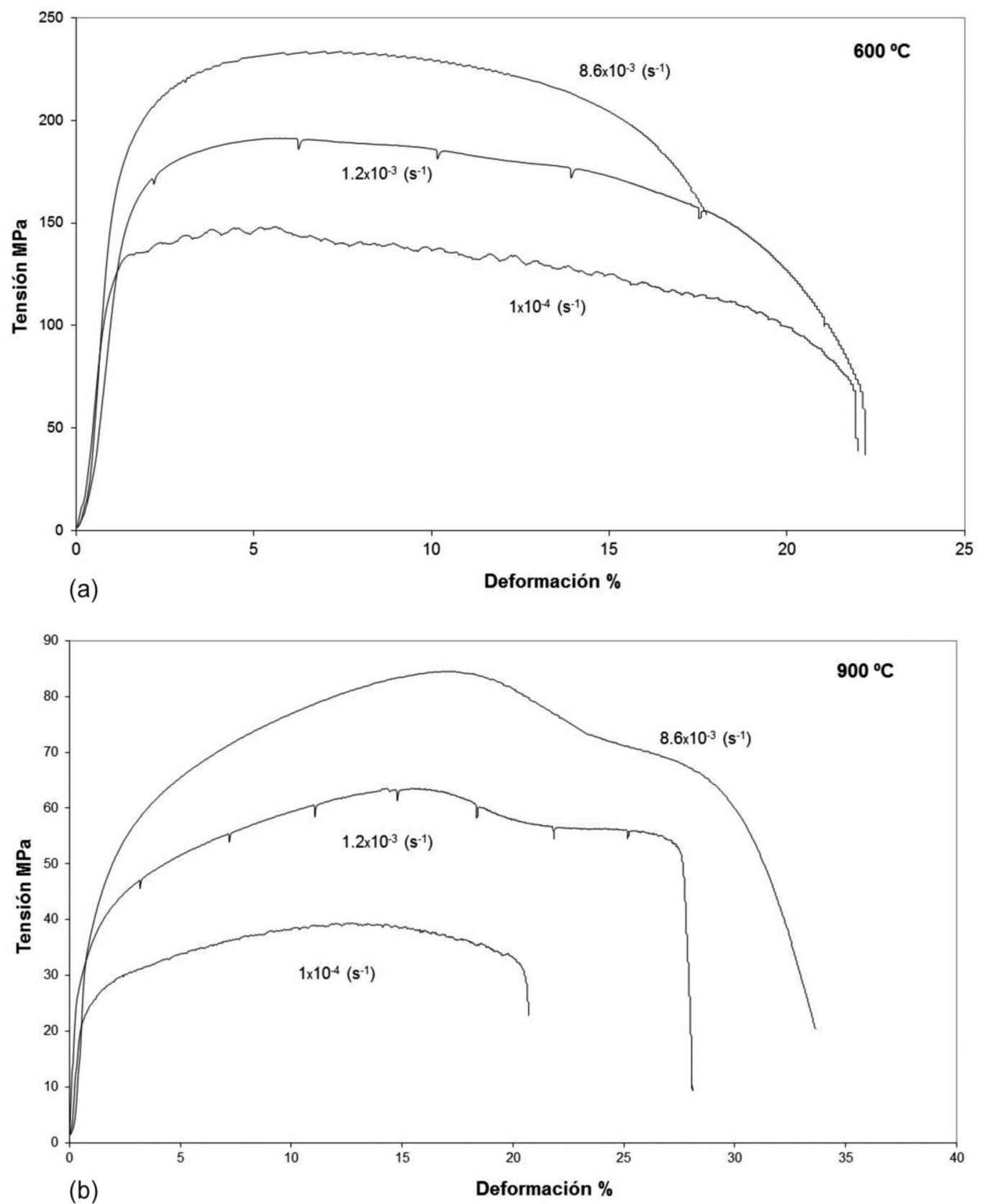

Figura 4. Ensayos de tracción (a) $600^{\circ} \mathrm{C}$; (b) $900^{\circ} \mathrm{C}$.

Figure 4. Tensile tests (a) $600^{\circ} \mathrm{C}$; (b) $900^{\circ} \mathrm{C}$.

metro A obtenido en este estudio es mayor que el valor obtenido por Urcola ${ }^{[25]}$ para aceros C-Mn, sin embargo, el valor obtenido para el parámetro n, es similar en ambos estudios.

A partir de la Ec. (6) y aplicando la Ec. (9) para el límite elástico, es posible calcular la tensión predicha por el modelo de Sellars necesaria para reducir el espesor del material. Además, esta tensión puede ser comparada con la tensión predicha mediante la medida de potencia a través de la Ec. (12), con $\omega=26 \mathrm{rpm}$. Finalmente la tensión promedio durante los ensayos de tracción para los diferentes valores de las velocidades de deformación, se estima usando la Ec. (11). Con este fin, para cada ensayo, se realiza el cálculo del área bajo la curva tensión-deformación, dividiendo luego este valor por la deformación introducida al material.

En la figura 6 se muestran de manera comparativa los valores de tensión predichos por los tres modelos. En el caso de la tensión predicha a partir de ensayos de tracción, se han utilizado los resultados correspondientes a la mayor velocidad de deformación, es 


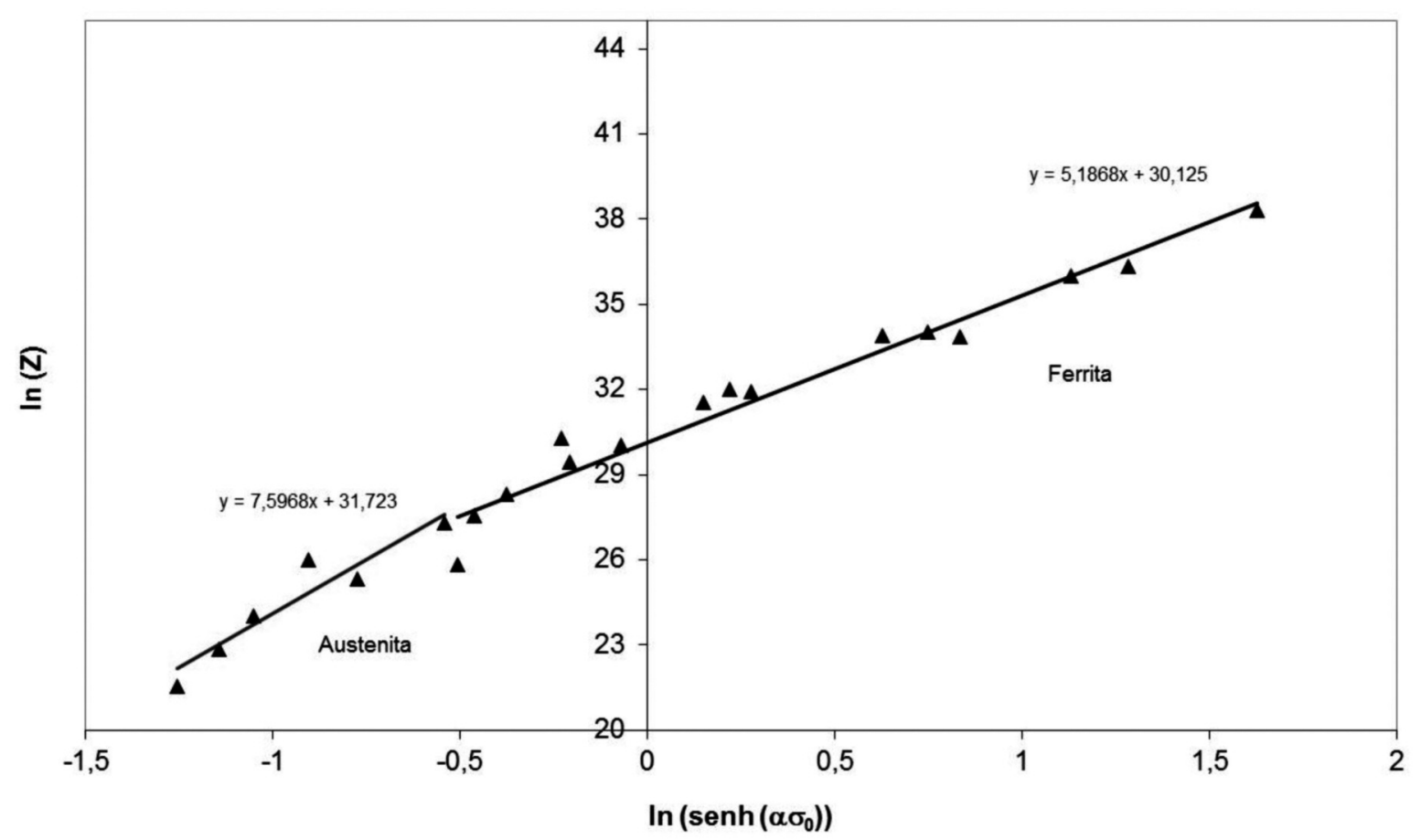

Figura 5. Relación entre el parámetro de Zener- Hollomon y $\ln \left[\operatorname{senh}\left(\alpha \sigma_{0}\right)\right]$.

Figure 5. Relation between the Zener-Hollomon parameter and In[senh $\left.\left(\alpha \sigma_{0}\right)\right]$.

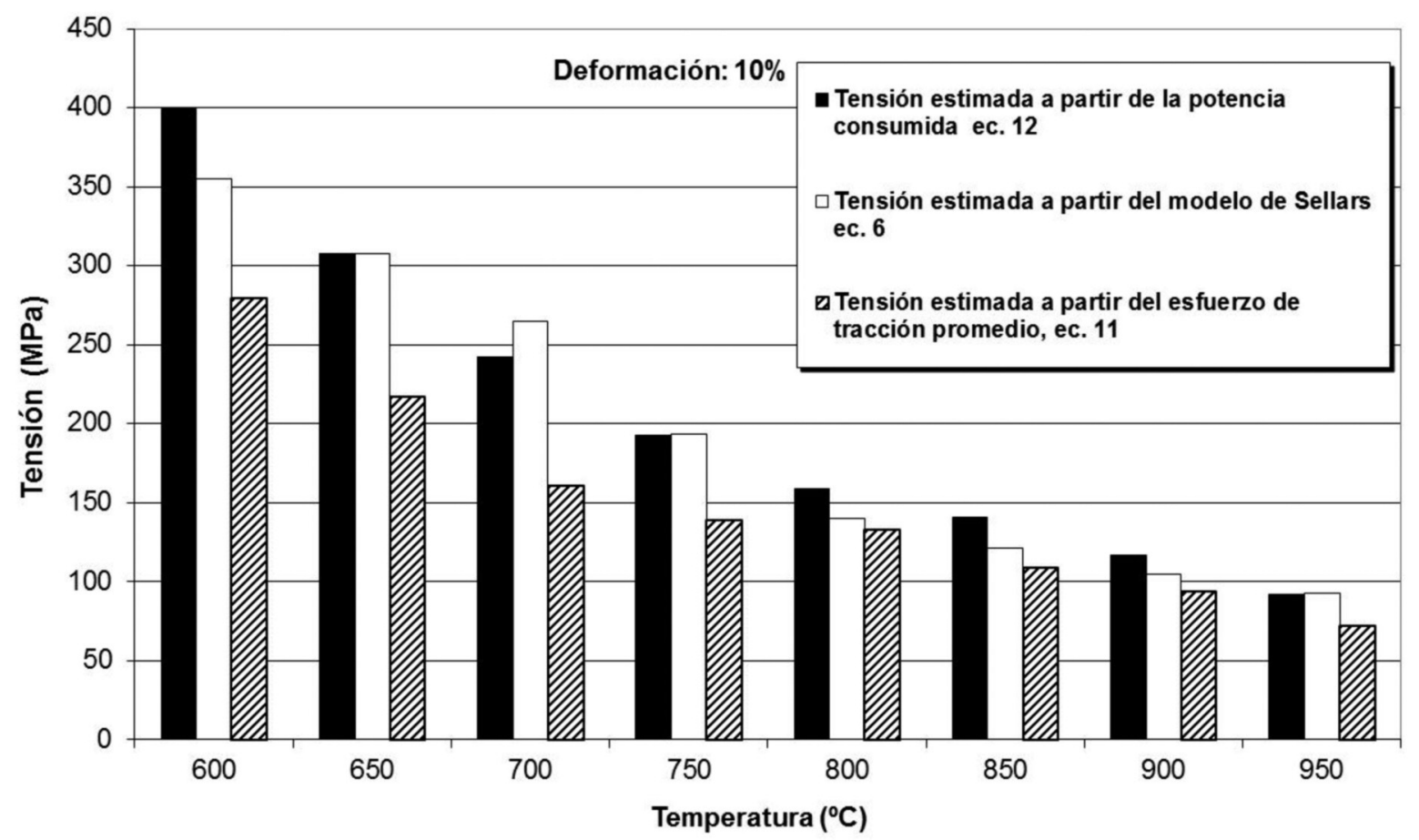

Figura 6. Tensión necesaria para laminar. A la izquierda: estimada a partir de la potencia consumida (Ec. (12)); centro: estimada a partir del modelo de Sellars (Ec. (6)); derecha: estimada a partir de la tensión de tracción promedio a velocidad de deformación de 8,6 $610^{-3}\left(\mathrm{~s}^{-1}\right)$ (Ec.(11)), para diferentes temperaturas, para $10 \%$ de deformación.

Figure 6. Stress necessary for rolling. Left: estimated from the power consumed (Eq. (12)); center: estimated from Sellars's model (Eq. (6)); right: estimated from the average tensile stress for a strain rate of $8.6 \times 10^{-3}\left(\mathrm{~s}^{-1}\right)$ (Eq. (11)), for different temperatures and $10 \%$ deformation. 
decir, $8,6 \times 10^{-3}\left(\mathrm{~s}^{-1}\right)$. Se aprecia una similitud aceptable entre los valores de tensión de laminación predichos por los tres métodos.

Para deformaciones totales por laminación de
20 y $30 \%$, los resultados se muestran en la figura 7 , donde se puede observar una buena correlación entre los valores predichos a partir de la Ec. (6) y aquéllos obtenidos mediante la Ec. (12).
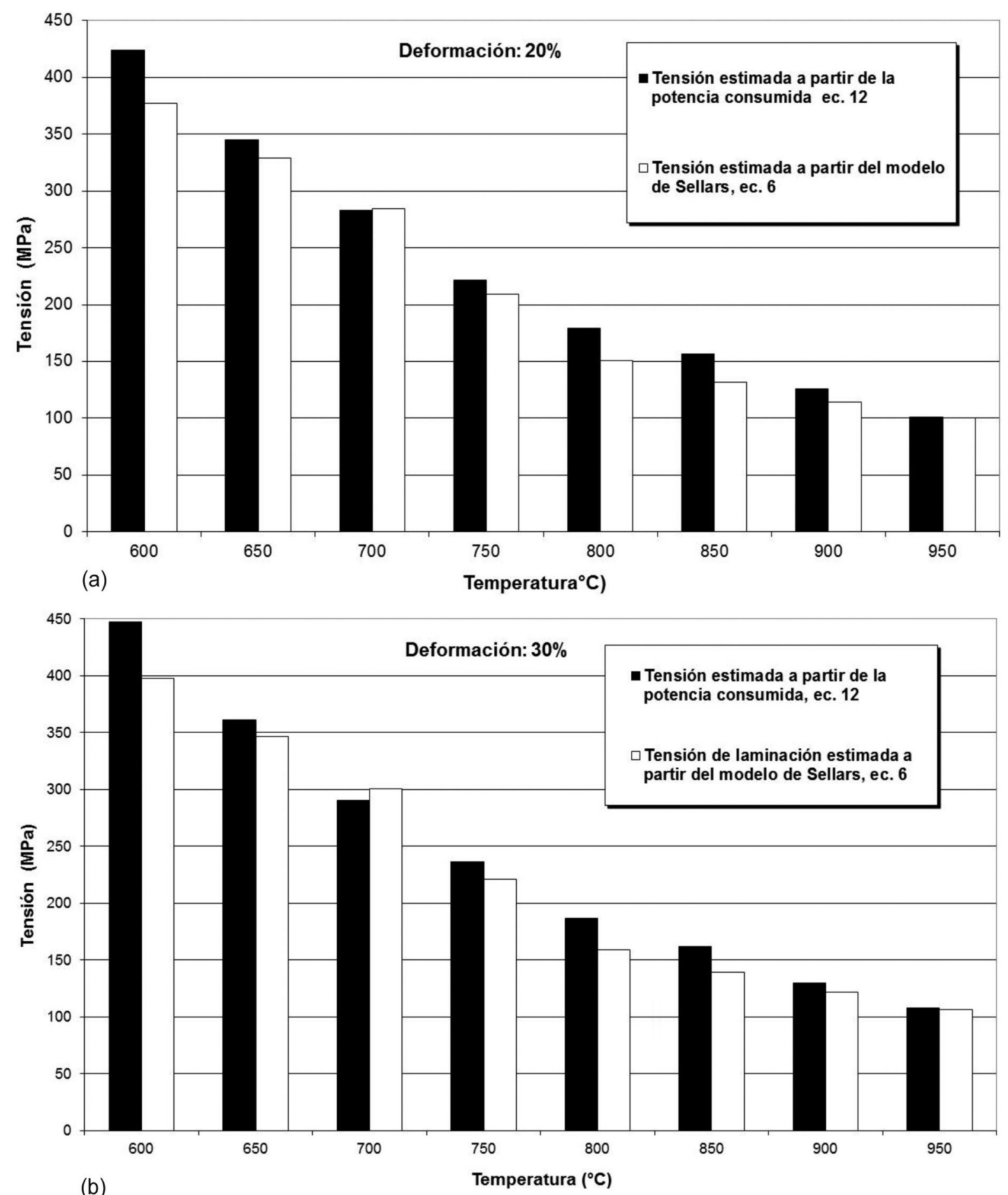

Figura 7. Tensión necesaria para laminar. A la izquierda: estimada a partir de la potencia consumida (Ec. (12)); centro: estimada a partir del modelo de Sellars (Ec. (6)). (a) $20 \%$ de deformación; (b) $30 \%$ de deformación.

Figure 7. Stress necessary for rolling. Left: estimated from the power consumed (Eq. (12)); center: estimated from Sellars's model (Eq. (6)); (a) $20 \%$ of strain; (b) $30 \%$ of strain. 
En esta figura, el valor de la tensión de tracción promedio no está representado, debido a que a deformaciones mayores del $10 \%$ aparece cuello sobre las probetas, por lo que la comparación no es válida.

En la figura 8, se muestra una comparación de los resultados obtenidos.
El valor de tensión de laminación obtenido mediante el modelo de Sellars (Ec. (6)), se muestra en el eje vertical, mientras que en el eje horizontal se muestra la tensión predicha a partir de la potencia consumida por el laminador, usando la Ec. (12). En la figura se grafica la línea recta $y=x$ para facilitar la comparación entre ambas tensio-

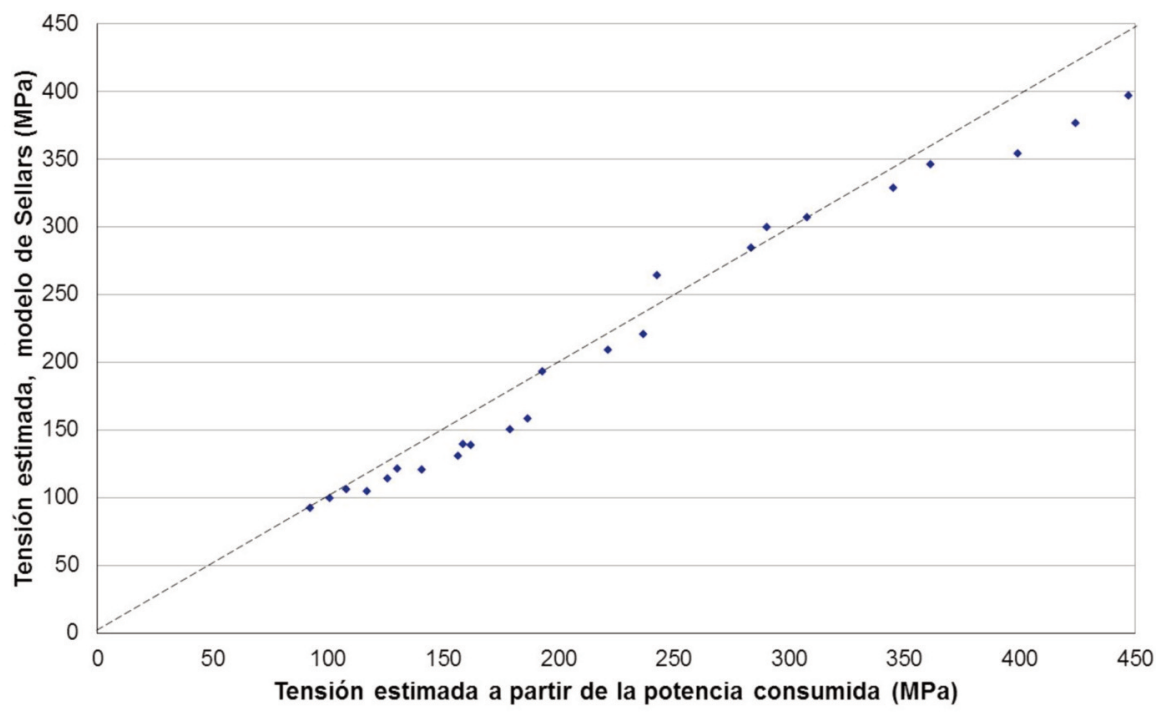

Figura 8. Comparación entre los valores de la tensión teórica estimada a partir del modelo de Sellars (eje vertical, Ec. (6)) y la tensión estimada a partir de la potencia consumida (eje horizontal, Ec. (12)).

\begin{abstract}
Figure 8. Comparison between the values of stress estimated from Sellars's model (vertical axis, Eq. (6)) and experimental stress estimated from consumed power (horizontal axis, Eq. (12)).
\end{abstract}

nes. Es posible observar una buena correlación entre ambos valores, concluyendo que ambas predicciones dan resultados similares, coherentes y razonables.

La diferencia promedio entre la tensión predicha por el modelo de Sellars (Ec. (6)) y la tensión predicha a partir de la potencia consumida usando la Ec. (12), es menor de 7,5 \% en el intervalo de temperaturas estudiado. La mayor diferencia entre ambas tensiones se produce a bajas temperaturas. De hecho, la diferencia promedio entre ambas tensiones es $7 \%$, si se consideran temperaturas mayores de $600^{\circ} \mathrm{C}$. En el caso de utilizar los valores de la tensión promedio obtenidos del ensayo de tracción para estimar la tensión de laminado en caliente, la diferencia promedio es de $25 \%$, demasiado grande para aplicaciones prácticas. Esta diferencia es esperable, debido a la gran diferencia entre las velocidades de deformación en un ensayo de tracción y en un proceso de laminación.
Se han comparado las predicciones de diferentes modelos para la obtención de la tensión de laminación, constatándose que la tensión necesaria para laminar en caliente el acero SAE 1025, puede ser estimada de manera satisfactoria usando el modelo propuesto por Sellars y el método de potencia de laminación. Fue posible estimar el valor de las tensiones de laminación, a partir de tensiones promedio provenientes de ensayos de tracción, para deformaciones pequeñas (inferiores a $10 \%$ ) y altas temperaturas (superiores a $800^{\circ} \mathrm{C}$ ), con una desviación menor del $20 \%$ respecto de los valores determinados experimentalmente.

\section{CONCLUSIONES}

- Se han comparado tres métodos para predecir la tensión de laminación: uno basado en los esfuerzos promedio en un ensayo de tracción; otro 
basado en el ajuste de Sellars que toma en cuenta el parámetro de Zener-Hollomon y otro basado en el cálculo de la tensión de laminación a partir de la medida de la potencia consumida por el laminador.

- El modelo de Sellars y el modelo basado en la estimación de la tensión de laminación a partir de la potencia consumida, muestran una buena concordancia entre sí, para las reducciones de espesor ensayadas.

- Los valores de las tensiones estimadas usando los valores promedios de las tensiones de tracción, son menores que los valores de las tensiones predichas por los otros métodos, debido a la menor velocidad de deformación usada en el ensayo de tracción.

\section{Agradecimientos}

Los autores desean agradecer a la Comisión Nacional de Investigación Científica y Tecnológica (CONICYT), proyecto $\mathrm{N}^{\circ} 1090311$ y a la Dirección de Investigaciones Científicas y Tecnológicas (DICYT), de la USACH. El autor R. Colás, desea agradecer al Consejo Nacional de Ciencia y Tecnología (CONACYT), México.

\section{REFERENCIAS}

[1] T. Lankford, The Making Shaping and Treating of Steel, 10 th Ed. USA., US Steel Co., 1985, pp. 1.069-1.070.

[2] W.L. Roberts, Hot Rolling of steels, CRC Press, USA, 1983, pp. 649-694.

[3] W.L. Roberts, Cold rolling of steels, Library Congress, New York, USA, 1978, pp. 447-489.

[4] J.G. Lenard, Primer for flat rolling, Elsevier Science Ltd., Amsterdam, 2007, pp. 36-98.

[5] G. Dieter, Mechanical Metallurgy, 3rd Ed. Mc. Graw-Hill, London, 1989, pp. 519-613.

[6] P.J. Wray, Ironmak. Steelmak. 14 (1987) 21-25.

[7] R.B. Sims, The calculation of roll forces and torque in hot rolling mills, Proceedings of the Institute of Mechanical Engineers, Vol. 168, 1954, pp. 191-198.

[8] H. Ford y J.M. Alexander, Journal of the Institute of Metals 92 (1964) 397-402.
[9] J. Kokado, N. Hatta, H. Takuda, S. Kikuchi y T. Hirabayashi, Steel Research 12 (1985) 619. 624.

[10] L. Béjar, C.A. Hernández y J.E. Mansilla, Rev. Latinoam. de Metalurgia y Materiales 21 (2001), 51-58.

[11] J.C. Gelin y O. Ghouati, J. Mater. Process. Tech. 34 (1994) 435-440.

[12] A. Gavrius, E. Massoni y J.L. Chenot, J. Mater. Process. Tech. 60 (1996) 447-454.

[13] B. Boyer y E. Massoni, Proc. of NUMIFORM 2001, Ed. K. Mori, A. Balkema, Toyohashi, 2001, pp. 281-284.

[14] R. Forestier, E. Massoni y Y. Chastel, J. Mater. Process. Tech. 125 (2002) 594-601.

[15] D. Szeliga, J. Gawad y M. Pietrzyk, Comp. Meth. Appl. Mech. Eng. 195 (2006) 6.7786.798.

[16] D. Szeliga, J. Gawad, M. Pietrzyk y R. Kuziak, Steel Res. Int. 76 (2005) 807-814.

[17] L. Papelux y J. Philippe, J. Mater. Process. Tech. (2002) 125-131.

[18] J. Schey, Tribology in metalworking: Friction, Lubrication and Wear, ASM, Metals Park, 1983, pp. 108-112.

[19] Y. Ohkomori, C. Sakae y Y. Murakami, 42th Mechanical Working and Steel Processing Conf. Proc., 38, ISS, Warrendale, 2000, p. 723.

[20] C. Sellars, Hot working and forming process Ed. por C. Sellars y M. Davies, The Metals Soc., London, 1980.

[21] R. Colás y M. Guerrero, Laminación en caliente y en frío de aceros: Características, propiedades y microestructuras, Aceros Estructructurales: Procesamiento, Manufactura y Propiedades, M.H. Staia y J.M. Cabrera, (eds.), CYTED, Barcelona, 2003, pp. 33-64.

[22] Standard Test Methods for Elevated Temperature Tension Tests of Metallic Materials, E-21, ASTM Standards, PA, 1989, pp. 184-190.

[23] H. Avendaño, Tesis Ing. Metal. y Magíster en Ciencias de la Ingeniería, USACH, 2005.

[24] R. Colás, J. Mater. Process. Tech. 62 (1996) 180-186.

[25] J. Urcola, M. Fuentes, Rev. Metal. Madrid 16 (1980) 337.

[26] W. Callister, Introducción a la Ciencia e Ingeniería de Materiales, Ed. Reverté, Barcelona, 1998, p. 305. 